\title{
Development of 30-kV Cc/Cs Correction Tandem System
}

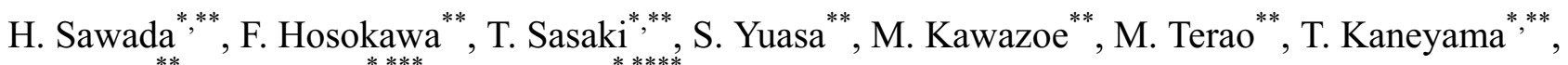 \\ Y. Kondo ${ }^{* *}$, K. Kimoto ${ }^{* * * *}$ K. Suenaga ${ }^{*}$ \\ *Japan Science and Technology Agency, CREST, 5, Sanbancho, Chiyoda-ku, Tokyo, 102-0075, \\ Japan \\ ** JEOL Ltd., 3-1-2 Musashino, Akishima, Tokyo, 196-8558, Japan \\ ${ }^{* * *}$ National Institute for Materials Science (NIMS), 1-1 Namiki, Tsukukba, Ibaraki 305-0044, Japan \\ ${ }^{* * * *}$ National Institute of Advanced Industrial Science and Technology (AIST), Tsukuba, 305-8565, \\ Japan
}

We are developing an atomic resolution low-voltage electron microscope in order to reduce electron-related irradiation damage on Triple C project (JST, 2006-2011) [1]. In addition to reduction of specimen damage, we demonstrated its effectiveness for high contrast and rich chemical signal that results from the larger inelastic scattering cross section at low acceleration voltage using Triple $\mathrm{C}$ microscope \# 1, which is equipped with Delta correctors and cold field emission gun working at 30-60 kV. [2,3]. The high contrast facilitates one's observation of light elements with an atomic resolution in TEM. For a TEM, resolution reduction by chromatic aberration is more severe than one for a STEM. Cc correction is a key to maintain resolution at the atomic level at extremely accerlating low-voltage. We found that a thick quadrupole field yields a concave lens by combination aberration [4]. This principle was applied to develop a Cc corrector for Triple C microscope \# 2 [5].

We designed and developed a Cs and Cc correction optical system, as shown in Fig. 1. Magnetic and electrostatic dodecapoles are used to make two-fold astigmatism field in the Cc corrector. The Cc corrector was mounted just below the objective lens. The Delta-type corrector [6] for a TEM was connected below the $\mathrm{Cc}$ corrector with transfer lenses to correct geometrical aberrations, resulting in a tandem $\mathrm{Cs} / \mathrm{Cc}$ correction system. Thus the tandem system, which is composed of two electrostatic and magnetic dodecapoles and three magenetic dodecapoles, was installed into the Triple C microscope \#2 [5]. The accelerating voltage of operation of the microscope is $30 \mathrm{kV}$. The operating applied voltage to the electrostatic dodecapole of Cc correction system is $4-5 \mathrm{kV}$. Cc correction was experimentally demonstrated, as previously reported [5].

Figure 2 shows diffractogram tableau, when geometrical and chromatic aberrations were corrected with the tandem $\mathrm{Cc} / \mathrm{Cs}$ correction system. No significant two-fold-symmetric pattern which is mainly originated from six-fold astigmatism after Cs correction, was detected in each diffactgram even in peripheral area of diffractgram tableau. It indicates that spherical aberration and six-fold astigmatism were corrected by the tandem system. And in the same diffractgrams at peripheral area, no decrease of intensity in radial direction, which is originated from chromatic aberration. And it indicates that chromatic aberration was corrected. Thus, we have confirmed the correcting actions of $\mathrm{Cs}, \mathrm{Cc}$ and other parasitic aberrations by the tandem system experimentally. This work is supported by the CREST project under Japan Science and Technology agency (JST).

\section{Reference}

[1] Triple C means Cs, Cc, and Carbon; a Cs and Cc correction technique is applied for structural and analytical studies of carbon-related materials.

[2] K. Suenaga, and M. Koshino, Nature 468 (2010) 1088. 
[3] T. Sasaki et al, J. Electron Microsc. 59 (2010) S7.

[4] F. Hosokawa, to be published.

[5] H. Sawada et al, Microscopy and Microanalysis. 16 (Suppl. 2) (2010) 116; T. Sasaki et al, IMC (2010) I2.2.

[6] H. Sawada et al, J. Electron Microsc. 58 (2009) 341.

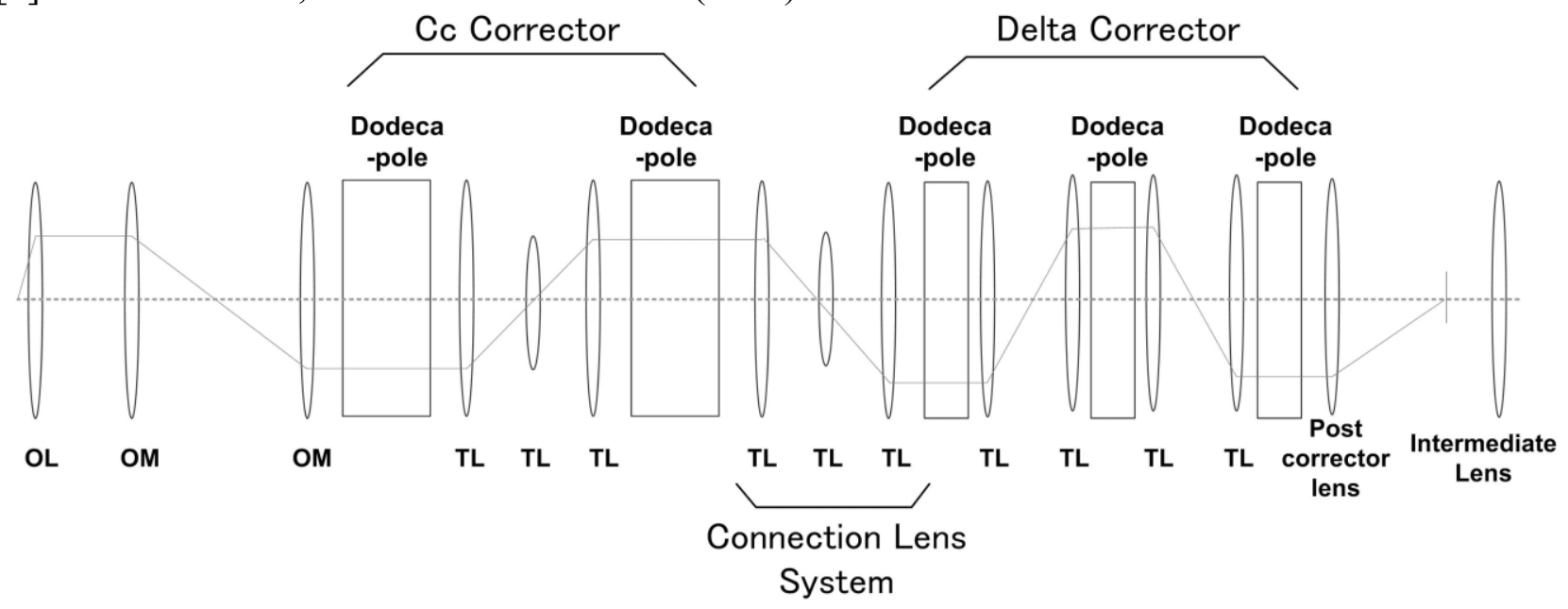

Fig. 1: Schematic of a tandem optical system for a TEM, which has a combination concave-lens-type Cc corrector and a Delta corrector.

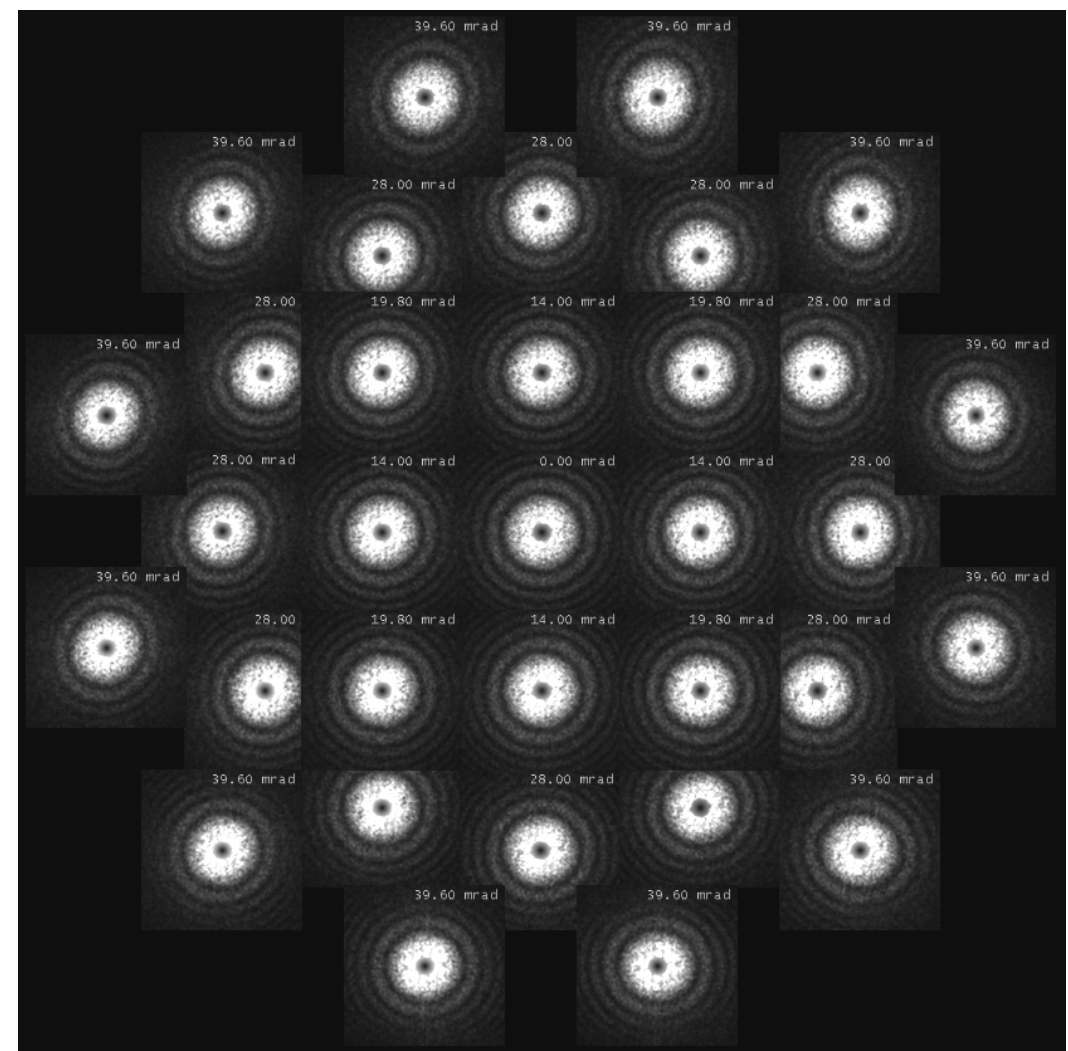

Fig. 2: Diffractogram tableau with Cc correction. Outer tilt angle is 39.6 mrad. The residual six-fold astigmatism was measured to be less than $0.1 \mathrm{~mm}$. 